\title{
ON THE ASYMPTOTIC FORMS OF THE SOLUTIONS OF ORDINARY LINEAR DIFFERENTIAL EQUATIONS OF THE THIRD ORDER IN A REGION CONTAINING A TURNING POINT $\left({ }^{(}\right)$
}

BY

RUDOLPH E. LANGER

1. Introduction. The subject of this paper is the derivation of asymptotic solutions for a class of differential equations of the type

$$
w^{\prime \prime \prime}+\lambda h_{1}(z, \lambda) w^{\prime \prime}+\lambda^{2} h_{2}(z, \lambda) w^{\prime}+\lambda^{8} h_{3}(z, \lambda) w=0,
$$

in which $\lambda$ is a large complex parameter, and the coefficients $h_{j}(z, \lambda)$ are expressible as power series in $1 / \lambda$,

$$
h_{j}(z, \lambda)=\sum_{n=0}^{\infty} \frac{h_{j, n}(z)}{\lambda^{n}},
$$$$
j=1,2,3 \text {. }
$$

Every such differential equation has associated with it a so-called characteristic or auxiliary equation

$$
\chi^{8}+h_{1,0}(z) \chi^{2}+h_{2,0}(z) \chi+h_{3,0}(z)=0 .
$$

This has three roots $\chi_{j}(z) ; j=1,2,3$. Differential equations (1.1) can be classified into categories having solutions of quite distinct functional forms upon a basis of the configuration of these roots $\chi_{j}(z)$ in the domain of $z$ under consideration. To some extent this can be seen even in the elementary case of an equation (1.1) with constant coefficients. For, such an equation is completely solved by the functions $e^{\lambda x_{j} \approx}$ if the roots $\chi_{j}$ are all simple, whereas this set is incomplete if multiplicities among the $\chi_{j}$ occur.

A substantially complete body of theory exists for the forms of the solutions of a differential equation (1.1) in a region of $z$ in which the roots $\chi_{j}(z)$ are everywhere distinct. [1]. Such is also the case if the $z$-region is one in which there are multiplicities among the roots $\chi_{j}(z)$, provided these multiplicities all maintain identically over the region $[2 ; 3]$. The matter stands differently for a region in which a multiplicity among the roots $\chi_{j}(z)$ occurs, but does so only at an isolated point. It is the purpose of the present paper to give a theory for a class of differential equations (1.1) in such a region of $z$.

The place of this theory, and its potential importance, can easily be made clear by referring to the analogous differential equation of the second order,

Presented to the Society, December 30, 1954; received by the editors July 1, 1954.

(1) The research reported in this paper was supported by the Office of Naval Research (Contract N7onr-28507). 


$$
w^{\prime \prime}+\lambda h_{1}(z, \lambda) w^{\prime}+\lambda^{2} h_{2}(z, \lambda) w=0 .
$$

This has the auxiliary equation $\chi^{2}+h_{1,0}(z) \chi+h_{2,0}(z)=0$, of which there are two roots, $\chi_{1}(z)$ and $\chi_{2}(z)$. The equation (1.4) is transformable by the removal of its first derivative term into the form

$$
u^{\prime \prime}+\left\{\lambda^{2} \phi^{2}(z)+\lambda h(z, \lambda)\right\} u=0,
$$

and in this $\phi^{2}(z)$, the coefficient of the highest power of $\lambda$, is found to be $-\left\{\chi_{1}(z)-\chi_{2}(z)\right\}^{2} / 4$. The existing theories referred to above are therefore those that apply to an equation (1.5) either in a $z$-region in which $\phi^{2}(z)$ has no zero, or in one over which $\phi^{2}(z)$ vanishes identically. The theory presently to be given, on the other hand, is an extension to a class of differential equations of the third order of that which is applicable to an equation (1.5) in a region in which $\phi^{2}(z)$ vanishes at just a point. Such a point has come to be known as a turning point, or a transition point.

The theory of differential equations (1.5) in regions containing a turning point has important applications in quantum mechanics. It also has important applications in other physical theories, among them that of microwave propagation [4]. Certain differential equations of the fourth and sixth orders with turning points are important in hydrodynamics, where they feature the onset of turbulence. They have been studied in that connection $[3 ; 5 ; 6 ; 7]$.

The presence of a turning point materially complicates the problem of describing a differential equation's solutions. For these solutions are then of different functional forms in different sub-regions of $z$, rather than of forms which persist over the whole region. As a rule their descriptions must be made in terms of branches of multiple-valued functions, even though the solutions themselves are single-valued.

The method of the present paper is based upon the use of a related differential equation. By this is meant a differential equation that fulfills two primary specifications, namely, (i), that its coefficients agree with those of the given equation to terms of some prescribed degree in $1 / \lambda$, and (ii), that it be explicitly solvable, namely that its solutions be known. The construction of such an equation is the objective of a major part of the analysis. It is followed by reasoning through which the forms of the solutions of the given equation are inferred from the known solutions of the related equation. This method was developed in connection with differential equations of the second order [8]. The present analysis extends it to a class of differential equations of higher order.

The hypotheses upon which the following discussion will be specifically based are the following.

1. The parameter $\lambda$ (complex) is large in absolute value. Thus $|\lambda|>N$, with some large constant $N$. 
2. The domain of the variable $z$ is a closed bounded region of the complex z-plane.

3. The series (1.2) have coefficients $h_{j, n}(z)$ that are analytic over the given $z$-region. Along with their term by term derivatives, these series are convergent when $|\lambda|>N$.

4. The roots of the auxiliary equation (1.3) are distinct over the given z-region, except at a single point (the turning point) where just two of them are equal.

5. At the turning point the zero of the discriminant of the auxiliary equation is of the first order.

By way of normalization, we shall suppose, as we may without loss of generality, that the origin of $z$ is at the turning point. We shall denote by $\chi_{1}(z)$ and $\chi_{2}(z)$ the roots of the equation (1.3) that coincide there. The remaining root $\chi_{3}(z)$ is therefore simple over the $z$-region. Some limitations upon the periphery of the $z$-region will have to be imposed in the course of the discussion. These cannot well be stated here, and are of only minor significance.

2. The normalization of the given differential equation. The analytic forms that are to be obtained for the representation of solutions of the equation (1.1) will involve certain factors that consist of polynomials in $1 / \lambda$ of a degree $(r-1)$, together with remainder terms of the order of $1 / \lambda r$. The polynomials will be determined, the remainder terms not so. The degree of explicitness of the representations will thus depend upon the integer $r$, a larger $r$ signifying a more explicit result. We may prescribe $r$, and shall henceforth suppose that to have been done. This particular significance will be reserved to $r$ throughout the discussion.

Let $\tau(z, \lambda)$ be a polynomial in $1 / \lambda$,

$$
\tau(z, \lambda)=\sum_{n=0}^{r} \frac{\tau_{n}(z)}{\lambda^{n}},
$$

of which the coefficients $\tau_{n}(z)$ are analytic, but otherwise, for the moment, unspecified. The substitution

$$
w=u \exp \left(\lambda \int_{0}^{z} \tau(z, \lambda) d z\right)
$$

transforms the differential equation (1.1) into

$$
u^{\prime \prime \prime}+2 \lambda p_{1}(z, \lambda) u^{\prime \prime}+\lambda^{2} p_{2}(z, \lambda) u^{\prime}+\lambda^{3} p_{3}(z, \lambda) u=0,
$$

with

$$
\begin{aligned}
2 p_{1} & =h_{1}+3 \tau \\
p_{2} & =h_{2}+2 h_{1} \tau+3 \tau^{2}+3 \tau^{\prime} / \lambda, \\
p_{3} & =h_{3}+h_{2} \tau+h_{1} \tau^{2}+\tau^{3}+\left(h_{1} \tau^{\prime}+3 \tau \tau^{\prime}\right) / \lambda+\tau^{\prime \prime} / \lambda^{2} .
\end{aligned}
$$


By setting

$$
L_{1}(u) \equiv u^{\prime \prime}+2 \lambda p_{1} u^{\prime}+\left\{\lambda^{2} p_{2}-2 \lambda p_{1}^{\prime}\right\} u,
$$

we may write (2.3) as

$$
L_{1}^{\prime}(u)+\lambda^{3}\left\{p_{3}-p_{2}^{\prime} / \lambda+2 p_{1}^{\prime \prime} / \lambda^{2}\right\} u=0 .
$$

The $p_{j}(z, \lambda)$ are evidently expressible as power series in $1 / \lambda$, namely

$$
p_{j}(z, \lambda)=\sum_{n=0}^{\infty} \frac{p_{j, n}(z)}{\lambda^{n}}, \quad j=1,2,3,
$$

and the same is therefore true of the function

$$
\left\{p_{3}-p_{2}^{\prime} / \lambda+2 p_{1}^{\prime \prime} / \lambda^{2}\right\} \text {. }
$$

We propose now to remove as large an initial segment from the series for (2.8) as may be possible by suitably specifying the coefficients $\tau_{n}(z)$ in (2.1).

The equation (1.3) has a simple root $\chi_{3}(z)$, and this root, because it is simple, is analytic. By taking

$$
\tau_{0}(z)=\chi_{3}(z),
$$

we make the leading term of $p_{3}$, and hence the leading term of (2.8), vanish. With this choice the leading term of $p_{2}$ is

$$
p_{2,0}(z)=h_{2,0}(z)+2 h_{1,0}(z) \chi_{3}(z)+3^{2} \chi_{3}(z),
$$

and this is nowhere zero because $\chi_{3}(z)$ is everywhere simple. Thus

$$
p_{2,0}(z) \neq 0
$$

over the given $z$-region. Now for $n=1,2, \cdots, r$, the coefficient of the term in $1 / \lambda^{n}$ in the series for (2.8) has the form

$$
\left\{p_{2,0}(z) \tau_{n}+f_{n}(z)\right\}
$$

where $f_{n}(z)$ is determined by the values of $\tau_{j}$ only for $j=0,1, \cdots,(n-1)$. It follows that we may successively specify the coefficients $\tau_{n}$ to make all these terms vanish. As a result, the function (2.8) is of the form $\lambda^{-r-1} p(z, \lambda)$, with $p(z, \lambda)$ a power series in $1 / \lambda$. The given differential equation is thus reducible to the form

$$
L_{1}^{\prime}(u)+\frac{p(z, \lambda)}{\lambda^{r-2}} u=0
$$

namely to

$$
\begin{aligned}
u^{\prime \prime \prime}+2 \lambda p_{1}(z, \lambda) u^{\prime \prime}+\lambda^{2} p_{2}(z, \lambda) u^{\prime}+\left\{\lambda^{2} p_{2}^{\prime}(z, \lambda)\right. & -2 \lambda p_{1}^{\prime \prime}(z, \lambda) \\
& \left.+p(z, \lambda) / \lambda^{r-2}\right\} u=0 .
\end{aligned}
$$


We shall refer to this as its normal form.

3. Miscellaneous matters. Since $\chi_{3}$ is a root, the equation (1.3) is divisible by $\left(\chi-x_{3}\right)$. The quotient, by use of the formulas $(2.4)$, is

$$
\left(x-\chi_{3}\right)^{2}+2 p_{1,0}(z)\left(x-\chi_{3}\right)+p_{2,0}(z)=0 .
$$

Hence

$$
\begin{aligned}
& \chi_{1}(z)=\chi_{3}(z)-p_{1,0}(z)+i \phi(z), \\
& \chi_{2}(z)=\chi_{3}(z)-p_{1,0}(z)-i \phi(z),
\end{aligned}
$$

with

$$
\phi^{2}(z)=p_{2,0}(z)-p_{1,0}^{2}(z) .
$$

At $z=0$ we have $\chi_{1}=\chi_{2}$, and $\chi_{1} \neq \chi_{3}$. It follows that $\phi(0)=0$, and

$$
p_{1,0}(0) \neq 0 \text {. }
$$

It also follows from (3.1) and (3.2) that

$$
\left(\chi_{1}-\chi_{2}\right)^{2}\left(\chi_{2}-\chi_{3}\right)^{2}\left(\chi_{3}-\chi_{1}\right)^{2}=-4 p_{2,0}^{2}(z) \phi^{2}(z) .
$$

Since this discriminant has, by hypothesis, a zero of the first order at $z=0$, it is to be concluded from (2.10) that the zero of $\phi^{2}(z)$ is simple.

Let

$$
\Phi(z)=\int_{0}^{z} \phi(z) d r, \quad \Psi(z)=\frac{\Phi^{1 / 6}(z)}{\phi^{1 / 2}(z)}, \quad \xi_{0}=\lambda \Phi(z) .
$$

The function $\phi(z)$ is of the form $z^{1 / 2} \phi_{1}(z)$ with $\phi_{1}(z)$ analytic and bounded from zero. Therefore $\Phi(z)$ is of the form $z^{3 / 2} \Phi_{1}(z)$, with $\Phi_{1}(z)$ analytic and bounded from zero in some neighborhood of $z=0$. We shall reduce the given $z$-region, if necessary, so that $\Phi_{1}(z)$ is bounded from zero within it. The function $\Psi(z)$ is now seen to be analytic (if defined to be continuous at $z=0$ ), and to be bounded and to have a bounded reciprocal.

By the substitution

$$
u=\exp \left(-\lambda \int_{0}^{z} p_{1}(z, \lambda) d z\right) U,
$$

the form (2.5) is replaced by one that lacks the first derivative term, thus

$$
L_{1}(u) \equiv \exp \left(-\lambda \int_{0}^{z} p_{1}(z, \lambda) d z\right) L_{0}(U)
$$

with

$$
L_{0}(U) \equiv U^{\prime \prime}+\left\{\lambda^{2}\left(p_{2}-p_{1}^{2}\right)-3 \lambda p_{1}^{\prime}\right\} U
$$


Because of (3.2) it is evident that

$$
L_{0}(U) \equiv U^{\prime \prime}+\left\{\lambda^{2} \phi^{2}(z)+\lambda \omega(z, \lambda)\right\} U,
$$

with $\omega(z, \lambda)$ a power series in $1 / \lambda$ namely

$$
\omega(z, \lambda)=\sum_{n=0}^{\infty} \frac{\omega_{n}(z)}{\lambda^{n}} .
$$

We observe from (3.4) that

$$
\frac{d}{d z}=\frac{\lambda^{2 / 3} \xi_{0}^{1 / 3}}{\Psi^{2}(z)} \frac{d}{d \xi_{0}} .
$$

4. The construction of a certain solvable differential equation of the second order. We propose now to find a pair of functions $\eta_{1}(z), \eta_{2}(z)$, that fulfill a differential equation approximating the equation $L_{0}(U)=0$. Under the relation (3.5) these will correspond to a pair of functions $y_{1}(z), y_{2}(z)$, that fulfill a differential equation approximating the equation $L_{1}(u)=0$. Later we shall associate with these a function $y_{0}(z)$ that is so determined as to make the differential equation of which $y_{j}(z), j=0,1,2$, are solutions approximate the equation $L_{1}^{\prime}(u)=0$, namely, by (2.11), approximate the given differential equation.

If $C_{1}$ and $C_{2}$ are any cylinder functions (Bessel functions) of the order $1 / 3$ so that

$$
\xi_{0}^{2} \frac{d^{2} C_{j}\left(\xi_{0}\right)}{d \xi_{0}^{2}}+\xi_{0} \frac{d C_{j}\left(\xi_{0}\right)}{d \xi_{0}}+\left[\xi_{0}^{2}-\frac{1}{9}\right] C_{j}\left(\xi_{0}\right)=0, \quad j=1,2,
$$

it is readily found, by the use of (3.9), that the functions

$$
v_{j}(z)=\Psi(z) \xi_{0}^{1 / 3} C_{j}\left(\xi_{0}\right)
$$

fulfill the equations

$$
v_{j}^{\prime}(z)+\left\{\lambda^{2} \phi^{2}(z)+\theta(z)\right\} v_{j}(z)=0,
$$

with

$$
\theta(z)=-\Psi^{\prime \prime}(z) / \Psi(z) .
$$

Thus the functions $v_{1}(z), v_{2}(z)$, fulfill a differential equation that approximates $L_{0}(U)=0$ to the extent that the most significant terms (in the highest power of $\lambda$ ) are the same in the two equations. We can do better with the functions

$$
\eta_{j}=A_{0} v_{j}+\frac{A_{1}}{\lambda} v_{j}^{\prime}, \quad j=1,2,
$$


if the coefficients $A_{0}(z, \lambda), A_{1}(z, \lambda)$ are appropriately determined. Preparatory to showing that, we shall derive the differential equation of which the functions (4.3) are solutions.

A differentiation of (4.3), followed by the elimination of $v_{j}^{\prime \prime}$ through the use of (4.2), yields

$$
\eta_{j}^{\prime}=\left[-\lambda \phi^{2} A_{1}+A_{0}^{\prime}-\theta A_{1} / \lambda\right] v_{j}+\left[A_{0}+A_{i}^{\prime} / \lambda\right] v_{j}^{\prime} .
$$

In a similar manner it is found that

$$
\begin{aligned}
\eta^{\prime \prime}= & {\left[-\lambda^{2} \phi^{2} A_{0}-2 \lambda \phi^{2} A_{1}^{\prime}-2 \lambda \phi \phi^{\prime} A_{1}+A_{0}^{\prime \prime}-\theta A_{0}-\frac{2 \theta A_{1}^{\prime}+\theta^{\prime} A_{1}}{\lambda}\right] v_{j} } \\
& +\left[-\lambda^{2} \phi^{2} A_{1}+2 \lambda A_{0}^{\prime}+A_{1}^{\prime \prime}-\theta A_{1}\right] \frac{v_{j}^{\prime}}{\lambda} .
\end{aligned}
$$

From (3.7) we therefore see that

$$
L_{0}\left(\eta_{j}\right)=S_{0} v_{j}^{\prime}-\lambda S_{1} v_{j},
$$

with

$$
S_{0}=2 A_{0}^{\prime}+\omega A_{1}+\frac{A_{1}^{\prime \prime}-\theta A_{1}}{\lambda}
$$

$$
S_{1}=2 \phi^{2} A_{1}^{\prime}+2 \phi \phi^{\prime} A_{1}-\omega A_{0}-\frac{A_{0}^{\prime \prime}-\theta A_{0}}{\lambda}+\frac{2 \theta A_{1}^{\prime}+\theta^{\prime} A_{1}}{\lambda^{2}} .
$$

By the elimination of $v_{j}$ and $v_{j}^{\prime}$ from the equations (4.3), (4.4), and (4.5) we obtain a relation that is fulfilled by $\eta_{j}$ for both $j=1$, and $j=2$. This is the differential equation

$$
\left|\begin{array}{ccc}
\eta & A_{0} & A_{1} / \lambda \\
\eta^{\prime} & -\lambda \phi^{2} A_{1}+A_{0}^{\prime}-\theta A_{1} / \lambda & A_{0}+A_{1}^{\prime} / \lambda \\
L_{0}(\eta) & -\lambda S_{1} & S_{0}
\end{array}\right|=0 .
$$

For the convenient expansion of this, let

$$
D(z, \lambda)=\left|\begin{array}{cc}
A_{0} & A_{1} / \lambda \\
-\lambda \phi^{2} A_{1}+A_{0}^{\prime}-\theta A_{1} / \lambda & A_{0}+A_{1}^{\prime} / \lambda
\end{array}\right|,
$$

It is then easily verified that

$$
D^{\prime}(z, \lambda)=\left|\begin{array}{cc}
A_{0} & A_{1} / \lambda \\
-\lambda S_{1} & S_{0}
\end{array}\right|
$$

and hence that the equation (4.7) is 


$$
D(z, \lambda) L_{0}(\eta)-D^{\prime}(z, \lambda) \eta^{\prime}+D_{*}(z, \lambda) \eta=0 .
$$

This is the differential equation of the functions (4.3). Its actual form depends, of course, upon the coefficients $A_{0}(z, \lambda)$ and $A_{1}(z, \lambda)$, which have thus far not been specified.

5. The determination of $A_{0}$ and $A_{1}$. Let $A_{0}$ and $A_{1}$ be polynomials in $1 / \lambda$, thus

$$
A_{j}(z, \lambda)=\sum_{n=0}^{r-1} \frac{a_{j, n}(z)}{\lambda^{n}}
$$$$
j=0,1 \text {, }
$$

with undetermined coefficients $a_{j, n}(z)$ that are analytic. The functions $S_{0}$ and $S_{1}$ as given by (4.6) are then evidently expressible as power series in $1 / \lambda$. We propose to remove from each of these series as large an initial segment as may be possible, by appropriately choosing the coefficients $a_{j, n}(z)$ in the polynomials $(5.1)\left({ }^{2}\right)$.

The leading terms of the series (4.6) are respectively the left-hand members of the equations

$$
\begin{aligned}
2 a_{0,0}^{\prime}+\omega_{0} a_{1,0} & =0, \\
2 \phi^{2} a_{1,0}^{\prime}+2 \phi \phi^{\prime} a_{1,0}-\omega_{0} a_{0,0} & =0 .
\end{aligned}
$$

We shall make these terms vanish by choosing $a_{0,0}$ and $a_{1,0}$ as a solution of the system of equations (5.2). To find this solution we multiply the equations respectively by $a_{0,0}$ and $a_{1,0}$, and then add them. The resulting equation is $\left(a_{0,0}^{2}+\phi^{2} a_{1,0}^{2}\right)^{\prime}=0$. We therefore take

$$
a_{0,0}^{2}+\phi^{2} a_{1,0}^{2}=1 .
$$

On substituting the value of $a_{1,0}$ from this into the first equation (5.2) and making the integration, we find that

$$
\begin{aligned}
& a_{0,0}=\cos \int_{0}^{z} \frac{\omega_{0}}{2 \phi} d z \\
& a_{1,0}=\frac{1}{\phi(z)} \sin \int_{0}^{z} \frac{\omega_{0}}{2 \phi} d z .
\end{aligned}
$$

The integral involved in these formulas is improper, because $\phi(z)$ vanishes like $z^{1 / 2}$ at $z=0$. It is, however, convergent, and the values (5.4) are seen to be in fact analytic in $z$ (with proper definition of $a_{1,0}(z)$ at $z=0$ ).

For $n=1,2, \cdots,(r-1)$, the coefficients of $1 / \lambda^{n}$ in the series (4.6) are of the forms of the left-hand members of the equations

(2) The method is essentially that which was given by the author, in a somewhat different formulation, in The asymptotic solutions of ordinary linear differential equations of the second order, with special reference to a turning point, Trans. Amer. Math. Soc. vol. 67 (1949) pp. 461490. 


$$
\begin{aligned}
2 a_{0, n}^{\prime}+\omega_{0} a_{1, n}-f_{0, n} & =0, \\
2 \phi^{2} a_{1, n}^{\prime}+2 \phi \phi^{\prime} a_{1, n}-\omega_{0} a_{0, n}-f_{1, n} & =0
\end{aligned}
$$

with $f_{0, n}$ and $f_{1, n}$ denoting expressions that involve the functions $a_{0, j}$ and $a_{1, j}$ only for $j=0,1,2, \cdots,(n-1)$. Thus (5.5) is a system of nonhomogeneous differential equations for $a_{0, n}$ and $a_{1, n}$. To solve it we proceed as follows. Multiply the equations (5.2) and (5.5) respectively by $\phi^{2} a_{1, n},-a_{0, n},-\phi^{2} a_{1,0}$, and $a_{0,0}$, and add them. Again, multiply them respectively by $a_{0, n}, a_{1, n}, a_{0,0}$, and $a_{1,0}$, and add them. The equations thus obtained are

$$
\begin{aligned}
2 \phi^{2}\left(a_{0,0} a_{1, n}-a_{1,0} a_{0, n}\right)^{\prime}+2 \phi \phi^{\prime}\left(a_{0,0} a_{1, n}-a_{1,0} a_{0, n}\right) & =f_{1, n} a_{0,0}-f_{0, n} \phi^{2} a_{1,0}, \\
2\left(\phi^{2} a_{1,0} a_{1, n}\right)^{\prime}+2\left(a_{0,0} a_{0, n}\right)^{\prime} & =f_{0, n} a_{0,0}+f_{1, n} a_{1,0},
\end{aligned}
$$

and these are easily integrated to give

$$
\begin{aligned}
a_{0,0} a_{1, n}-a_{1,0} a_{0, n} & =\frac{1}{\phi(z)} \int_{0}^{z} \frac{f_{1, n} a_{0,0}-f_{0, n} \phi^{2} a_{1,0}}{2 \phi} d z, \\
\phi^{2} a_{1,0} a_{1, n}+a_{0,0} a_{0, n} & =\int_{0}^{z} \frac{f_{0, n} a_{0,0}+f_{1, n} a_{1,0}}{2} d z .
\end{aligned}
$$

As a system in the unknowns $a_{0, n}$ and $a_{1, n}$ these equations have, by (5.3), a determinant whose value is 1 . They may, therefore, be solved to give

$$
\begin{aligned}
& a_{0, n}=a_{0,0} \int_{0}^{z} \frac{f_{0, n} a_{0,0}+f_{1, n} a_{1,0}}{2} d z-\phi a_{1,0} \int_{0}^{z} \frac{f_{1, n} a_{0,0}-f_{0, n} \phi^{2} a_{1,0}}{2 \phi} d z, \\
& a_{1, n}=a_{1,0} \int_{0}^{z} \frac{f_{0, n} a_{0,0}+f_{1, n} a_{1,0}}{2} d z+\frac{a_{0,0}}{\phi} \int_{0}^{z} \frac{f_{1, n} a_{0,0}-f_{0, n} \phi^{2} a_{1,0}}{2 \phi} d z .
\end{aligned}
$$

These values are seen to be analytic despite the improper integrals in terms of which they are expressed. By the evaluations (5.6) the terms in the successive powers of $1 / \lambda$ in the functions (4.6) are removed, and these functions are thus reduced to be of the order of $1 / \lambda^{r}$.

With the polynomials $A_{0}$ and $A_{1}$ thus determined, the expression $D(z, \lambda)$, as given by (4.8), is a polynomial in $1 / \lambda$ the initial term of which is 1 . The equation (4.10) may therefore be divided by $D(z, \lambda)$ when $|\lambda|$ is sufficiently large. Under the substitution

$$
\eta=\exp \left(\lambda \int_{0}^{z} p_{1}(z, \lambda) d z\right) D^{1 / 2}(z, \lambda) y,
$$

the equation assumes the form

$$
y^{\prime \prime}+2 \lambda p_{1} y^{\prime}+\left\{\lambda^{2} p_{2}-2 \lambda p_{1}^{\prime}-q(z, \lambda) / \lambda^{r-1}\right\} y=0,
$$

with 


$$
q(z, \lambda) / \lambda^{-1}=(3 / 4) D^{\prime 2} / D^{2}-D^{\prime \prime} / D+D_{*} / D \text {. }
$$

Since, with $S_{0}$ and $S_{1}$ of the order of $\lambda^{-r}$, the values $D^{\prime}$ and $D_{*}$ are respectively of the orders of $\lambda^{-r}$ and $\lambda^{-r+1}$, it is clear that $q(z, \lambda)$ is a power series in $1 / \lambda$.

The equation (5.8) has the explicitly known solutions

$$
\begin{array}{r}
y_{j}=\exp \left(-\lambda \int_{0}^{z} p_{1}(z, \lambda) d z\right) D^{-1 / 2}(z, \lambda)\left\{A_{0}(z, \lambda) v_{j}+\frac{1}{\lambda} A_{1}(z, \lambda) v_{j}^{\prime}\right\} \\
j=1,2 .
\end{array}
$$

By setting

$$
L(y) \equiv y^{\prime \prime}+2 \lambda p_{1} y^{\prime}+\left\{\lambda^{2} p_{2}-2 \lambda p_{1}^{\prime}-q / \lambda^{r-1}\right\} y,
$$

we may write it $L(y)=0$. In an obvious sense the operator $L$ approximates the operator $L_{1}$ of $(2.5)$.

6. The "related" differential equation. We shall now determine a function $y_{0}(z)$ in such a way as to make the three functions $y_{j}(z), j=0,1,2$, be a fundamental set of solutions of a differential equation that approximates the given differential equation.

Let $y_{0}(z, \lambda)$ be the polynomial in $1 / \lambda$ given by the formula

$$
y_{0}(z, \lambda)=\sum_{n=0}^{r-1} \frac{y_{0, n}(z)}{\lambda^{n}}
$$

with coefficients $y_{0, n}(z)$ that remain to be specified. By direct formal substitution into the form $(5: 10)$ it is found then that

$$
L\left(y_{0}\right)=\lambda^{2} p_{2,0} y_{0,0}+\sum_{n=1}^{r-1} \frac{p_{2,0} y_{0, n}+f_{n}}{\lambda^{n-2}}+\sum_{n=r}^{\infty} \frac{f_{n}}{\lambda^{n-2}},
$$

with each $f_{n}$ denoting an expression that depends upon the functions $y_{0, j}(z)$ only for $j<n$. Since $p_{2,0}(z) \neq 0$, by (2.10), we may choose the functions $y_{0, n}(z)$, for successive values of $n$, so that

$$
\begin{aligned}
p_{2,0}(z) y_{0,0}(z) & =1, \\
p_{2,0}(z) y_{0, n}(z)+f_{n}(z) & =0, \quad n=1,2, \cdots,(r-1) .
\end{aligned}
$$

These functions $y_{0, n}(z)$ are analytic, and give to (6.2) the form

$$
L\left(y_{0}\right)=\lambda^{2}\left\{1+\frac{g_{0}(z, \lambda)}{\lambda^{r}}\right\}
$$

with $g_{0}(z, \lambda)$ a power series in $1 / \lambda$. By virtue of this, then,

$$
L^{\prime}\left(y_{0}\right) / L\left(y_{0}\right)=g(z, \lambda) / \lambda^{r},
$$

$g(z, \lambda)$ also being a power series in $1 / \lambda$ with coefficients that are analytic in $z$.

The differential equation 


$$
L^{\prime}(y)-\left(g(z, \lambda) / \lambda^{r}\right) L(y)=0
$$

is obviously solved by the function $y_{0}(z)$, and by any solution of $L(y)=0$. Therefore it is solved by $y_{1}(z)$ and $y_{2}(z)$. To show that this set of solutions is complete we consider the Wronskian

$$
W_{0,1,2}(z)=\left|\begin{array}{lll}
y_{0}(z) & y_{1}(z) & y_{2}(z) \\
y_{0}^{\prime}(z) & y_{1}^{\prime}(z) & y_{2}^{\prime}(z) \\
y_{0}^{\prime \prime}(z) & y_{1}^{\prime \prime}(z) & y_{2}^{\prime \prime}(z)
\end{array}\right| .
$$

In this we may replace each element $y_{j}^{\prime \prime}$ by the respective $L\left(y_{j}\right)$, since that amounts only to adding multiples of other rows to a certain row of a determinant. Then since $L\left(y_{1}\right)=0$, and $L\left(y_{2}\right)=0$, it follows that

$$
W_{0,1,2}(z)=L\left(y_{0}\right) W_{1,2}(z) \text {. }
$$

If $y_{1}$ and $y_{2}$ are linearly independent, which, by (5.9), is so if $v_{1}$ and $v_{2}$ are independent, the right-hand member of (6.7) is different from zero over the $z$-region. The equation (6.5) is therefore completely solved by $y_{j}(z), j=0,1,2$.

Now by (2.5) and (5.10)

$$
L_{1}(u)=L(u)+\frac{q}{\lambda^{r-1}} u .
$$

The given differential equation (2.11) is thus expressible as

$$
L^{\prime}(u)=-\frac{p}{\lambda^{r-2}} u-\frac{(q u)^{\prime}}{\lambda^{r-1}},
$$

and this we may write as

$$
L^{\prime}(u)-\frac{g(z, \lambda)}{\lambda^{r}} L(u)=Q(u, z)
$$

with

$$
Q(u, z)=\frac{\lambda^{2} p u+\lambda(q u)^{\prime}+g(z, \lambda) L(u)}{-\lambda^{r}} .
$$

The reduced form of (6.8), namely the equation obtained from it by replacing the right-hand member by zero, is the solvable equation (6.5). It is evident that these equations have coefficients that are the same to terms in $1 / \lambda^{r-2}$. We shall call (6.5) the related differential equation.

7. The forms of some solutions of the related equation. Of the solutions $y_{0}, y_{1}$, and $y_{2}$, of the related equation (6.5), $y_{0}(z)$ has been given explicitly by the formula (6.1). The others may be variously chosen as independent solutions of the equation (5.8). It is important to consider the forms of these, since they will be found to be typical of the solutions of the given differential 
equation when $|\lambda|>N$. The related differential equation is, after all, merely a particular equation of the type we are considering.

From (4.1), with the cylinder functions $C_{j}$ taken successively to be the Bessel functions $J_{-1 / 3}$ and $J_{1 / 8}$ we obtain the two functions $\left(^{(3)}\right.$

$$
v_{*, j}=\Psi(z) \xi_{0}^{1 / 3} J_{\mp 1 / 3}\left(\xi_{0}\right) .
$$

These functions can be differentiated. By use of the formula (3.7), together with the known relations, cf. [9, p. 46],

$$
\frac{d}{d \xi_{0}}\left\{\xi_{0}^{\nu} J_{ \pm \nu}\left(\xi_{0}\right)\right\}= \pm \xi_{0}^{\nu} J_{ \pm(\nu-1)}\left(\xi_{0}\right)
$$

it is found that

$$
v_{*, j}^{\prime}(z)=\Psi^{\prime}(z) \xi_{0}^{1 / 3} J_{\mp 1 / 3}\left(\xi_{0}\right) \mp \frac{\lambda^{2 / 3}}{\Psi(z)} \xi_{0}^{2 / 3} J_{ \pm 2 / 3}\left(\xi_{0}\right) .
$$

The relation (5.9) now yields as solutions $y_{1}$ and $y_{2}$ the particular ones $y_{*, j}$, where

$$
\begin{aligned}
& y_{*, j}(z)=\exp \left(-\lambda \int_{0}^{z} p_{1}(z, \lambda) d z\right)\left\{E_{0}(z) \xi_{0}^{1 / 3} J_{\mp 1 / 3}\left(\xi_{0}\right) \mp \frac{E_{1}(z)}{\lambda^{1 / 3}} \xi_{0}^{2 / 3} J_{ \pm 2 / 3}\left(\xi_{0}\right)\right\}, \\
& y_{*, j}^{\prime}(z)=\exp \left(-\lambda \int_{0}^{z} p_{1}(z, \lambda) d z\right) \lambda\left\{G_{0}(z) \xi_{0}^{1 / 3} J_{\mp 1 / 3}\left(\xi_{0}\right) \mp \frac{G_{1}(z)}{\lambda^{1 / 3}} \xi_{0}^{2 / 3} J_{ \pm 2 / 3}\left(\xi_{0}\right)\right\},
\end{aligned}
$$

with

$$
\begin{aligned}
& E_{0}=\frac{\lambda A_{0} \Psi+A_{1} \Psi^{\prime}}{\lambda D^{1 / 2}}, \\
& E_{1}=\frac{A_{1}}{\Psi D^{1 / 2}}, \\
& G_{0}=-p_{1} E_{0}-\Psi^{2} \phi^{2} E_{1}+E_{0}^{\prime} / \lambda \\
& G_{1}=-p_{1} E_{1}+\frac{E_{0}}{\Psi^{2}}+E_{1}^{\prime} / \lambda .
\end{aligned}
$$

The functions of $\xi_{0}$ that are involved in (7.3) are given by the familiar expansions

$$
\xi_{0}^{\nu} J_{ \pm \nu}\left(\xi_{0}\right)=\sum_{n=0}^{\infty} \frac{(-1)^{n} 2^{\nu}}{n ! \Gamma(n+1 \pm \nu)}\left(\frac{\xi_{0}}{2}\right)^{2 n+\nu \pm \nu}
$$

(3) It is to be understood in all that follows that when a double sign and the index $j$ occur in a formula or equation, the upper sign is to be taken with $j=1$ and the lower sign with $j=2$. 
These are useful especially when $z$ is in the immediate neighborhood of the turning point, namely when $\left|\xi_{0}\right|$ is moderate or small. In particular, we find from (7.3) and (7.5) the evaluations

$$
\begin{array}{rlrl}
y_{*, 1}(0) & =\frac{2^{1 / 3} E_{0}(0)}{\Gamma(2 / 3)}, & y_{*, 2}(0) & =\frac{2^{2 / 3} E_{1}(0)}{\lambda^{1 / 3} \Gamma(1 / 3)}, \\
y_{*, 1}^{\prime}(0)=\frac{2^{1 / 3} \lambda G_{0}(0)}{\Gamma(2 / 3)}, & y_{*, 2}^{\prime}(0)=\frac{2^{2 / 8} \lambda^{2 / 3} G_{1}(0)}{\Gamma(1 / 3)} .
\end{array}
$$

Now it can be verified from (7.4) and (4.8) that

$$
\left|\begin{array}{cc}
E_{0}(z) & E_{1}(z) \\
G_{0}(z) & G_{1}(z)
\end{array}\right| \equiv 1
$$

From this, and the fact that $\Gamma(1 / 3) \Gamma(2 / 3)=2 \pi / 3^{1 / 2}$, it follows that the Wronskian of $y_{*, 1}$ and $y_{*, 2}$ has at $z=0$ the value $3^{1 / 2} \lambda^{2 / 8} / \pi$. Since the differential equation for these functions is (5.8), the formula for the Wronskian for general $z$ is

$$
W\left(y_{*, 1}, y_{*, 2} ; z\right)=\frac{3^{1 / 2} \lambda^{2 / 3}}{\pi} \exp \left(-2 \lambda \int_{0}^{z} p_{1}(z, \lambda) d z\right) .
$$

8. The forms of some other solutions of the related equation. The solutions $y_{*, j}$ described in $\S 7$ have advantageous forms when $\left|\xi_{0}\right|$ is small or moderate. They are, however, not of the optimum simplicity when $\left|\xi_{0}\right|$ is large. In fact that advantage does not attach to any single pair of solutions, except in suitable sub-regions of $\lambda$ and $z$. We therefore divide the $\xi_{0}$-region, and therewith the z-region, into parts, within each of which an especially associated pair of solutions is distinctively simple. For the representation of these solutions we shall use the Hankel functions $H_{\nu}^{(1)}$ (Bessel functions of the third kind, cf. [9, p. 73]).

For each integer $k$, a sector $\boldsymbol{\Xi}_{k}$ of the complex $\xi_{0}$-plane is defined by the relations

$$
\Xi_{k}:(k-1) \pi<\arg \xi_{0}<(k+1) \pi .
$$

In the $z$-domain each $\boldsymbol{\Xi}_{k}$ corresponds to a sub-region having the origin upon its boundary. Its location beyond that depends upon $\lambda$, since $\xi_{0}$ does so. Let $z$ be in such a sub-region. The integer $k$ is thereby determined. With this, and with $j$ taking the values 1 and 2 , we shall use the abbreviations

$$
\begin{aligned}
& \sigma=e^{-k \pi i}, \\
& \mu=\left\{\begin{array}{l}
1, \text { if } j+k \text { is odd, } \\
2, \text { if } j+k \text { is even. }
\end{array}\right.
\end{aligned}
$$

In accordance with the relation (4.1), with suitable choices of the cylinder functions, we now set 


$$
v_{k, j}(z)=(\sigma \pi / 2)^{1 / 2} \exp \left( \pm \frac{5 \pi i \sigma}{12}\right) \Psi(z) \xi_{0}^{1 / 3} H_{1 / 3}^{(\mu)}\left(\xi_{0} e^{-k \pi i}\right) .
$$

These functions can be differentiated. By the use of (3.9), and the known relations, cf. $[9$, p. 74$]$,

$$
\frac{d}{d \xi}\left\{\xi^{\nu} H_{\nu}^{(\mu)}(\xi)\right\}=\xi^{\prime} H_{\nu-1}^{(\mu)}(\xi),
$$

it is found that

$$
\begin{gathered}
v_{k, j}^{\prime}(z)=\left(\frac{\sigma \pi}{2}\right)^{1 / 2} \exp \left( \pm \frac{5 \pi i \sigma}{12}\right)\left\{\Psi^{\prime}(z) \xi_{0}^{1 / 3} H_{1 / 3}^{(\mu)}\left(\xi_{0} e^{-k \pi i}\right)\right. \\
\left.+\frac{\sigma \lambda^{2 / 3}}{\Psi(z)} \xi_{0}^{2 / 3} H_{-2 / 3}^{(\mu)}\left(\xi_{0} e^{-k \pi i}\right)\right\} .
\end{gathered}
$$

The formulas (5.9) and (7.4) therewith yield as solutions $y_{j}$ the particular ones

$$
\begin{aligned}
y_{k, j}(z)= & \left(\frac{\sigma \pi}{2}\right)^{1 / 2} \exp \left( \pm \frac{5 \pi i \sigma}{12}-\lambda \int_{0}^{z} p_{1}(z, \lambda) d z\right)\left\{E_{0}(z) \xi_{0}^{1 / 3} H_{1 / 3}^{(\mu)}\left(\xi_{0} e^{-k \pi i}\right)\right. \\
& \left.+\frac{\sigma E_{1}(z)}{\lambda^{1 / 3}} \xi_{0}^{2 / 3} H_{-2 / 3}^{(\mu)}\left(\xi_{0} e^{-k \pi i}\right)\right\},
\end{aligned}
$$

$$
\begin{aligned}
y_{k, j}^{\prime}(z)= & \left(\frac{\sigma \pi}{2}\right)^{1 / 2} \exp \left( \pm \frac{5 \pi \sigma i}{12}-\lambda \int_{0}^{z} p_{1}(z, \lambda) d z\right) \lambda\left\{G_{0}(z) \xi_{0}^{1 / 3} H_{1 / 3}^{(\mu)}\left(\xi_{0} e^{-k \pi i}\right)\right. \\
& \left.+\frac{\sigma G_{1}(z)}{\lambda^{1 / 3}} \xi_{0}^{2 / 3} H_{-2 / 3}^{(\mu)}\left(\xi_{0} e^{-k \pi i}\right)\right\} .
\end{aligned}
$$

The Hankel functions admit of the asymptotic representations

$$
H_{\nu}^{(j)}(\zeta) \sim\left(\frac{2}{\pi \zeta}\right)^{1 / 2} \exp \left( \pm i\left[\zeta-\frac{\nu \pi}{2}-\frac{\pi}{4}\right]\right) \sum_{n=0}^{\infty} \frac{(\nu, n)}{(\mp 2 i \zeta)^{n}}
$$

with

$$
(\nu, n)=\frac{\Gamma(\nu+n+1 / 2)}{n ! \Gamma(\nu-n+1 / 2)},
$$

when $|\zeta|$ is large, provided $-\pi<\arg \zeta<\pi$, cf. [9, p. 201]. These conditions upon $\zeta$ are fulfilled by $\xi_{0} e^{-k \pi i}$ when $\xi_{0}$ is in the sector (8.1) and $\left|\xi_{0}\right|$ is large. The forms (8.6) then give

$$
\left(\frac{\sigma \pi}{2}\right)^{1 / 2} \exp \left( \pm \frac{5 \pi i \sigma}{12}\right) H_{1 / 3}^{(\mu)}\left(\xi_{0} e^{-k \pi i}\right) \sim \xi_{0}^{-1 / 2} e^{ \pm i \xi_{0}} \sum_{n=0}^{\infty} \frac{(1 / 3, n)}{\left(\mp 2 i \xi_{0}\right)^{n}}
$$




$$
\sigma\left(\frac{\sigma \pi}{2}\right)^{1 / 2} \exp \left( \pm \frac{5 \pi i \sigma}{12}\right) H_{-2 / 3}^{(\mu)}\left(\xi_{0} e^{-k \pi i}\right) \sim \pm i \xi_{0}^{-1 / 2} e^{ \pm i \xi_{0}} \sum_{n=0}^{\infty} \frac{(-2 / 3, n)}{\left(\mp 2 i \xi_{0}\right)^{n}}
$$

Hence, by (8.5),

$$
y_{k, j}(z) \sim \xi_{0}^{-1 / 6} \exp \left( \pm i \xi_{0}-\lambda \int_{0}^{z} p_{1}(z, \lambda) d z\right) \Theta_{0, j}\left(z, \xi_{0}, \lambda\right),
$$

$$
y_{k, j}^{\prime}(z) \sim \lambda \xi_{0}^{-1 / 6} \exp \left( \pm i \xi_{0}-\lambda \int_{0}^{z} p_{1}(z, \lambda) d z\right) \Theta_{1, j}\left(z, \xi_{0}, \lambda\right),
$$

with

$$
\begin{aligned}
& \Theta_{0, j} \equiv E_{0}(z) \sum_{n=0}^{\infty} \frac{(1 / 3, n)}{\left(\mp 2 i \xi_{0}\right)^{n}} \pm i \Phi^{1 / 3}(z) E_{1}(z) \sum_{n=0}^{\infty} \frac{(-2 / 3, n)}{\left(\mp 2 i \xi_{0}\right)^{n}} \\
& \Theta_{1, j} \equiv G_{0}(z) \sum_{n=0}^{\infty} \frac{(1 / 3, n)}{\left(\mp 2 i \xi_{0}\right)^{n}} \pm i \Phi^{1 / 3}(z) G_{1}(z) \sum_{n=0}^{\infty} \frac{(-2 / 3, n)}{\left(\mp 2 i \xi_{0}\right)^{n}} .
\end{aligned}
$$

The related differential equation therefore has in each sector (8.1) a pair of solutions having the asymptotic forms (8.7).

It is known that

$$
H_{1 / 3}^{(\mu)}\left(\xi_{0} e^{-k \pi i}\right)=\frac{2}{3^{1 / 2} \sigma^{1 / 3}} e^{\mp \sigma \pi i / 12}\left\{J_{-1 / 3}\left(\xi_{0}\right)-\sigma^{2 / 3} e^{F_{\sigma \pi i / 3}} J_{1 / 3}\left(\xi_{0}\right)\right\},
$$

cf. $[9$, p. 75]. The solutions (7.3) and (8.5) are thus connected by the relations

$$
y_{k, j}(z)=\left(\frac{2 \pi}{3}\right)^{1 / 2} \sigma^{1 / 6} e^{\mp i \sigma / 12}\left\{y_{*, 1}(z)-\sigma^{2 / 3} e^{\mp \pi i \sigma / 3} y_{*, 2}(z)\right\},
$$

of which the inverses are

$$
\begin{aligned}
& y_{*, 1}(z)=\frac{\sigma^{5 / 6}}{i(2 \pi)^{1 / 2}}\left\{e^{5 \pi i \sigma / 12} y_{k, 1}(z)-e^{-5 \pi i \sigma / 12} y_{k, 2}(z)\right\}, \\
& y_{*, 2}(z)=\frac{\sigma^{1 / 6}}{i(2 \pi)^{1 / 2}}\left\{e^{\pi i \sigma / 12} y_{k, 1}(z)-e^{-\pi i \sigma / 12} y_{k, 2}(z)\right\} .
\end{aligned}
$$

On the basis of these we readily infer from (7.8) that

$$
W\left(y_{k, 1}, y_{k, 2} ; z\right)=-2 i \lambda^{2 / 8} \exp \left(-2 \lambda \int_{0}^{z} p_{1}(z, \lambda) d z\right),
$$

and from (6.7) and (6.3) that

$$
\text { (8.12) } W\left(y_{0}, y_{k, 1}, y_{k, 2} ; z\right)=-2 i \lambda^{8 / 8} \exp \left(-2 \lambda \int_{0}^{z} p_{1}(z, \lambda) d z\right)\left\{1+\frac{g_{0}(z, \lambda)}{\lambda^{r}}\right\} \text {. }
$$


Finally, the values which the $y_{k, j}(z)$ take on at $z=0$ are obtainable from (8.9) and (7.6).

9. Abbreviations, and some simple appraisals. The explicitness of the formulas of $\S 7$ and $\S 8$ extends effectively to the order of $1 / \lambda^{r}$. This will insure results that also have that degree of explicitness. For the derivation of these results, however, less exact, and therefore simpler, formulas will to a considerable extent suffice.

By way of abbreviations, let

$$
P(z)=\int_{0}^{z} p_{1,0}(z) d z,
$$

and set

$$
\xi_{j}= \pm \xi_{0}+i \lambda P(z)
$$$$
j=1,2 \text {. }
$$

Also let the symbol $\xi_{0}^{8}(z)$ be interpreted thus

$$
\xi_{0}^{\prime}(z)= \begin{cases}1, & \text { when }\left|\xi_{0}(z)\right| \leqq N, \\ \xi_{0}^{1 / 6}(z), & \text { when }\left|\xi_{0}(z)\right|>N,\end{cases}
$$

and let the relations

$$
\begin{array}{ll}
\Omega_{0}(z) & =\frac{W\left(y_{k, 1}, y_{k, 2} ; z\right)}{W\left(y_{0}, y_{k, 1}, y_{k, 2} ; z\right)}, \\
\Omega_{j}(z) & =\frac{\mp W\left(y_{0}, y_{k, 3-j} ; z\right)}{W\left(y_{0}, y_{k, 1}, y_{k, 2} ; z\right)},
\end{array}
$$

define their left-hand members. The constant $N$ shall always be understood to be large enough to make the asymptotic representations (8.7) usable. The precise value of $N$ is never significant. Finally, let $B$ be adopted as a generic symbol to signify a function that is bounded when $|\lambda|>N$. This boundedness shall be uniform as to any other variables that may be involved, as when the symbol used is $B(z, \lambda)$ or $B(z, t, \lambda)$, and relative to such other variables any function $B$ shall be understood to be analytic, or at least so in piecewise fashion over certain sub-regions. Since the region of $z$ has been taken to be bounded and closed, any function that is analytic in it is also bounded.

When $\xi_{0}$ is in the sector $\Xi_{k}$ and $\left|\xi_{0}\right|>N$, the representations (8.7), (8.8) show that

$$
\begin{aligned}
& y_{k, j}(z)=\xi_{0}^{-1 / 6} \exp \left( \pm i \xi_{0}-\lambda \int_{0}^{z} p_{1}(z, \lambda) d z\right) B(z, \lambda), \\
& y_{k, j}^{\prime}(z)=\lambda \xi_{0}^{-1 / 6} \exp \left( \pm i \xi_{0}-\lambda \int_{0}^{z} p_{1}(z, \lambda) d z\right) B(z, \lambda) .
\end{aligned}
$$


On the other hand, since the functions $e^{ \pm i \xi_{0}}$ and $\xi_{0}^{\nu} H_{ \pm \nu}^{(j)}\left(\xi_{0}\right)$ with $\nu>0$ are bounded when $\left|\xi_{0}\right| \leqq N$, whereas for all $z$

$$
\exp \left( \pm \lambda \int_{0}^{z} p_{1}(z, \lambda) d z\right)=e^{ \pm \lambda P(z)} B(z, \lambda)
$$

we see from (8.5) that

$$
\begin{aligned}
& y_{k, j}(z)=\exp \left( \pm i \xi_{0}-\lambda P(z)\right) B(z, \lambda), \\
& y_{k, j}^{\prime}(z)=\lambda \cdot \exp \left( \pm i \xi_{0}-\lambda P(z)\right) B(z, \lambda), \text { when }\left|\xi_{0}\right| \leqq N .
\end{aligned}
$$

We may combine these appraisals by the use of the relations (9.2) and (9.3) into the forms

$$
\begin{aligned}
& y_{k, j}(z)=\frac{e^{i \xi_{j}} B(z, \lambda)}{\xi_{0}^{s}(z)}, \\
& y_{k, j}^{\prime}(z)=\frac{\lambda e^{i \xi_{j}} B(z, \lambda)}{\xi_{0}^{s}(z)}, \text { when } \xi_{0} \text { is in } z_{k} ; \quad j=1,2 .
\end{aligned}
$$

For use along with these, the formula (6.1) gives

$$
y_{0}(z)=B(z, \lambda), \quad y_{0}^{\prime}(z)=B(z, \lambda) .
$$

The relations (6.3), (8.11), and (8.12) yield

$$
\begin{aligned}
W\left(y_{k, 1}, y_{k, 2} ; z\right) & =\lambda^{2 / 3} e^{-2 \lambda P(z)} B(z, \lambda), \\
\frac{1}{W\left(y_{0}, y_{k, 1}, y_{k, 2} ; z\right)} & =\lambda^{-8 / 3} e^{2 \lambda P(z)} B(z, \lambda),
\end{aligned}
$$

whereas (9.5) and (9.6) show that

$$
W\left(y_{0}, y_{k, j} ; z\right)=\frac{\lambda e^{i \xi_{i} B(z, \lambda)}}{\xi_{0}^{s}(z)} .
$$

We thus find for the functions (9.4) the appraisals

$$
\begin{array}{ll}
\Omega_{0}(z)=\frac{B(z, \lambda)}{\lambda^{2}}, & \\
\Omega_{j}(z)=\frac{e^{-i \xi_{i}} B(z, \lambda)}{\lambda^{5 / 3 \xi_{0}^{\prime}}(z)}, & j=1,2 .
\end{array}
$$

Finally, with $Q$ defined by (6.9), the evaluations (6.3), (9.5), and (9.6), to gether with the fact that $L\left(y_{k, j}\right)=0$, lead to the relations

$$
Q\left(y_{0}, z\right)=\frac{B(z, \lambda)}{\lambda^{r-2}}, \quad Q\left(y_{k, j}, z\right)=\frac{e^{i \xi_{i}} B(z, \lambda)}{\lambda^{r-2} \xi_{0}^{s}(z)} .
$$


10. The given and related equations. With the forms of the solutions of the related equation now at hand, it remains to infer from them the forms of the solutions of the given equation. We shall show how this may be done.

If, in the equation (6.8), $u$ is thought of as any particular solution $u(z)$, the right-hand member of the equation is accordingly a specific function of $z$, namely $Q(u(z), z)$. Upon denoting this by $f(z)$, the function $u(z)$ in question is seen to be also a solution of the non-homogeneous equation

$$
L^{\prime}(u)-\frac{g(z, \lambda)}{\lambda^{r}} L(u)=f(z) .
$$

Now the reduced companion of this is the related equation (6.5). Hence $u(z)$ is expressible in terms of the functions $y_{0}, y_{k, 1}$, and $y_{k, 2}$, thus

$$
\begin{aligned}
u(z)= & c_{0} y_{0}(z)+c_{1} y_{k, 1}(z)+c_{2} y_{k, 2}(z) \\
& +\int^{z}\left|\begin{array}{ccc}
y_{0}(z) & y_{k, 1}(z) & y_{k, 2}(z) \\
y_{0}(t) & y_{k, 1}(t) & y_{k, 2}(t) \\
y_{0}^{\prime}(t) & y_{k, 1}^{\prime}(t) & y_{k, 2}^{\prime}(t)
\end{array}\right| \frac{f(t) d t}{W\left(y_{0}, y_{k, 1}, y_{k, 2} ; t\right)},
\end{aligned}
$$

namely, by virtue of (9.4), by a formula

$$
u(z)=\sum_{n=0}^{2} c_{n} y_{k, n}(z)+\sum_{n=0}^{2} y_{k, n}(z) \int^{z} \Omega_{n}(t) f(t) d t,
$$

wherein $y_{k, 0} \equiv y_{0}$. The lower limits of integration, which have not been inserted in (10.1), may be taken at arbitrary fixed points of the $z$-region. When that has been done, the constants $c_{0}, c_{1}, c_{2}$ are determined for the function $u(z)$. The formula (10.1) may be differentiated twice as though the integrals were independent of $z$, since the sum of terms that arise from differentiation of these integrals vanishes. Thus

$$
\begin{aligned}
& u^{\prime}(z)=\sum_{n=0}^{2} c_{n} y_{k, n}^{\prime}(z)+\sum_{n=0}^{2} y_{k, n}^{\prime}(z) \int^{z} \Omega_{n}(t) f(t) d t, \\
& u^{\prime \prime}(z)=\sum_{n=0}^{2} c_{n} y_{k, n}^{\prime \prime}(z)+\sum_{n=0}^{2} y_{k, n}^{\prime \prime}(z) \int^{z} \Omega_{n}(t) f(t) d t .
\end{aligned}
$$

From (10.1) and (10.2) we may construct the expression $Q(u, z)$, and since this is precisely what was denoted by $f(z)$ we find thus that

$$
f(z)=\sum_{n=0}^{2} c_{n} Q\left(y_{k, n}, z\right)+\sum_{n=0}^{2} Q\left(y_{k, n}, z\right) \int^{z} \Omega_{n}(t) f(t) d t .
$$

This deduction was based upon the assumption of a particular solution $u(z)$. Its character is therefore heuristic. We shall use it merely as a guide to the following deduction $a b$ initio, which is effectively its reverse. 
With a suitably chosen set of lower limits of integration and constants $c_{n}$, the integral equation (10.3) will be shown to have a solution $f(z)$. The form of this solution will be determined, and in terms of it a function $u(z)$ will be defined by the formula (10.1). The formulas (10.2) follow, and if $\left.Q^{\prime} u, z\right)$ is formed from these, it is found, by a comparison of the result with (10.3), that $Q(u, z)$ is precisely $f(z)$. In the relations $(10.1)$ and $(10.2)$ we may therefore replace $f(t)$ by $Q(u, t)$. If the expression $L(u)$ is then formed from (10.1) and (10.2) it is found that

$$
L(u)=c_{0} L\left(y_{0}\right)+L\left(y_{0}\right) \int^{z} \Omega_{0}(t) Q(u, t) d t,
$$

whereas it follows from this, since $y_{0}$ fulfills the equation (6.5), that

$$
L^{\prime}(u)-\frac{g(z, \lambda)}{\lambda^{r}} L(u)=L\left(y_{0}\right) \Omega_{0}(z) Q(u, z) .
$$

But this is the given differential equation, by (6.7) and (9.4). The function $u(z)$ as thus defined by $(10.1)$ is therefore a solution of the given equation. From (10.1) its form will be determinable.

11. Associated regions of $\lambda$ and $z$. Since $\phi^{2}(z)$ has a simple zero at the turning point, the functions $\phi(z)$ and $\Phi(z)$ are double-valued. To account for this we shall henceforth consider the z-region to be a two-sheeted domain, namely a Riemann surface with a branch point at its origin. The formulas

$$
\begin{aligned}
& \xi_{0}=\lambda \Phi(z), \\
& \xi_{1}=i \lambda\{P(z)-i \Phi(z)\}, \\
& \xi_{2}=i \lambda\{P(z)+i \Phi(z)\},
\end{aligned}
$$

which have already been set forth as (3.4) and (9.2), define $\xi_{0}, \xi_{1}$, and $\xi_{2}$ as complex variables, and determine maps of the $z$-region upon respective $\xi_{j}-$ regions. These latter are likewise Riemann surfaces with branch points at their origins, the $\xi_{0}$-region being three-sheeted because near $z=0$ the function $\Phi$ has the character of $z^{3 / 2}$, and the regions of $\xi_{1}$ and $\xi_{2}$ being two-sheeted because $P$ has the character of $\left(z^{1 / 2}\right)^{2}$.

In each $\xi_{j}$-region let $R\left(\xi_{j}\right)$ denote the largest sub-region that is star-shaped with respect to the origin. These regions $R\left(\xi_{j}\right)$, for $j=0,1,2$, have images in the domain of $z$ under the mappings (11.1), and these images have a common part (intersection). This common part we shall denote by $R(z)$. It may be noted, in particular, that any sufficiently small neighborhood of $z=0$ is in $R(z)$.

Since each of the variables (11.1) depends upon $\lambda$, each region $R\left(\xi_{j}\right)$ likewise does so. The dependence is, however, simple, for any change in $\lambda$ merely changes the scale of $R\left(\xi_{j}\right)$ in the ratio of the change in $|\lambda|$, and rotates $R\left(\xi_{j}\right)$ 
by the amount of the change in $\arg \lambda$. The region $R(z)$ is clearly independent of $\lambda$.

Let $\boldsymbol{z}^{(c)}$ be any chosen point of $R(z)$, and $\lambda^{(c)}$ any chosen value of $\lambda$ for which $\left|\lambda^{(c)}\right|$ is large. These values together determine $\xi_{j}^{(c)}$ for each $j$. Now for any $j$ we can enclose $\xi_{j}^{(c)}$ within a polygon that lies in $R\left(\xi_{j}\right)$, that is convex, that has no horizontal side, and that also encloses the origin. This polygon, since it encloses a branch point, has a perimeter that extends several times around this point. It therefore has a number of uppermost vertices and a number of lowermost ones, namely certain vertices at which the ordinate is a relative maximum or minimum. With one such uppermost vertex, and with one such lowermost one, the point $\xi_{j}^{(c)}$ can be connected by respective straight line segments. We shall denote these vertices by $\xi_{j, M}$ and $\xi_{j, m}$ respectively. Now under rotations of the polygon which are suitably limited in range, the vertex $\xi_{j, M}$ will retain its property of being an uppermost one, and $\xi_{j, m}$ will retain its property of being a lowermost one. Such a range of $\arg \lambda$ defines a sector of the $\lambda$-plane which we shall designate by $\Lambda$. The point $\lambda^{(c)}$ is in this sector.

In $R\left(\xi_{j}\right)$ we now define a sub-region $R^{(c)}\left(\xi_{j}\right)$ by the following specifications: (a) it contains the point $\xi_{j}^{(c)}$; (b) every point of it can, for every $\lambda$ in $\Lambda$, be connected with $\xi_{j, M}$ by a curve along which the ordinate is nondecreasing; and (c) every point of it can, for every $\lambda$ in $\Lambda$, be connected with $\xi_{j, m}$ by a curve along which the ordinate is nonincreasing. The regions $R^{(c)}\left(\xi_{j}\right)$, for $j=0,1,2$, have images under the mappings (11.1), and these images have a common part. We denote this common part by $R_{\Delta}(z)$, and we shall say that the sector $\Lambda$ and the region $R_{\Delta}(z)$ are associated regions.

We have shown that any pair of points $z$ and $\lambda$ of which the former is in $R(z)$ can be enclosed in associated regions. Any region $R^{(c)}\left(\xi_{j}\right)$ is easily seen to be a sector bounded by two lines on which arg $\xi_{j}$ is constant. Also any region $R^{(c)}\left(\xi_{0}\right)$ necessarily lies in some sector $\Xi_{k}$. Every region $R_{\Delta}(z)$ therefore has the point $z=0$ upon its boundary, and is one within which a relation (8.1) maintains. Finally, each pair of regions $\Lambda$ and $R_{\Delta}(z)$ has associated with it a set of points, namely the points $z_{j, M}$ and $z_{j, m}, j=0,1,2$, that are the images of the vertices $\xi_{j, M}$ and $\xi_{j, m}$. These points are fixed, namely do not vary with $\lambda$, so long as $\lambda$ remains in the sector $\Lambda$. The points $z_{j, M}$ and $z_{j, m}$ are in $R(z)$, but not necessarily in $R_{\Delta}(z)$.

12. Paths of integration. When $z$ and $\lambda$ are confined to a pair of associated regions, we shall choose the lower limits of integration of the relations (10.3) and (10.1) at the points $z_{j, M}$ and $z_{j, m}$ thereby determined. The paths of integration from these points to $z$ may be chosen at pleasure, since the integrands are analytic. We shall choose them so as to facilitate appraisals of the integrals.

With $j$ as any one of the indices $0,1,2$, and with $\mu$ standing for either $M$ or $m$, let $\Gamma_{j, z}$ denote the path from $z_{j, \mu}$ to $z$. We describe this path with 
reference to its appearance in $R\left(\xi_{j}\right)$ as follows. It is the straight segment from $\xi_{j, \mu}$ to $\xi_{j}$, provided this lies wholly in $R\left(\xi_{j}\right)$; otherwise it is the broken line consisting of the segments from $\xi_{j, \mu}$ to the origin, and from there to $\xi_{j}$. On every such path the ordinate of $\xi_{j}$, and hence the real part of $i \xi_{j}$, varies monotonically. Thus

$$
\begin{aligned}
& e^{-i \xi_{j}(z)+i \xi_{j}(t)} \text { is bounded when } t \text { is on } \Gamma_{j, M}, \text { and } \\
& e^{i \xi_{j}(z)-i \xi_{j}(t)} \text { is bounded when } t \text { is on } \Gamma_{j, m} .
\end{aligned}
$$

We may now establish the following lemma.

Lemma. An integral of the form

$$
\int_{\Gamma_{j, \mu}}\left|B(z, t, \lambda) \frac{\lambda^{1 / 8} d t}{\left[\xi_{0}^{z}(t)\right]^{2}}\right|
$$

is bounded uniformly in $z$, when $|\lambda|>N$.

Consider this first when $j=0$. Then, by (3.9),

$$
\frac{\lambda^{1 / 8} d t}{\left[\xi_{0}^{s}(t)\right]^{2}}=\left\{\Psi^{2}(t)\left[\frac{\xi_{0}^{1 / 6}(t)}{\xi_{0}^{s}(t)}\right]^{2}\right\} \frac{d \xi_{0}}{\lambda^{1 / 8} \xi_{0}^{2 / 8}},
$$

and since the function within the brace is bounded the integral (12.2) is a bounded multiple of

$$
\int_{\Gamma_{0, \mu}}\left|\frac{d \xi_{0}}{\lambda^{1 / 8} \xi_{0}^{2 / 8}}\right|
$$

If the path $\Gamma_{0, \mu}$ passes through the origin of $R\left(\xi_{0}\right)$, it consists of segments upon which $\left|d \xi_{0}\right|= \pm d\left|\xi_{0}\right|$. Hence (12.3) is no greater than

$$
\left(3 /|\lambda|^{1 / 8}\right)\left\{\left|\xi_{0, \mu}\right|^{1 / 8}+\left|\xi_{0}\right|^{1 / 8}\right\},
$$

which is bounded. If the path does not pass through the origin, let $\xi_{*, 0}$ be its nearest point to the origin. Then since $d \xi_{0}=d\left(\xi_{0}-\xi_{*, 0}\right)$ and along the path $\left|\xi_{0}\right|>\left|\xi_{0}-\xi_{*, 0}\right|$, the integral (12.3) is less than

$$
\int_{\Gamma_{0, \mu}}\left|\frac{d\left(\xi_{0}-\xi_{*, 0}\right)}{\lambda^{1 / 8}\left(\xi_{0}-\xi_{*, 0}\right)^{1 / 3}}\right|
$$

Inasmuch as the path is now one on which $\left|d\left(\xi_{0}-\xi_{*, 0}\right)\right|= \pm d\left|\xi_{0}-\xi_{*, 0}\right|$, we may again reach the previous conclusion.

When $j=1,2$, the relation (11.1) yields

$$
d t=\frac{d \xi_{j}}{i \lambda\left\{p_{1,0}(t) \mp i \phi(t)\right\}},
$$


and by (3.2) this is

$$
d t=\frac{\left\{p_{1,0}(t) \pm i \phi(t)\right\}}{i p_{2,0}(t)} \frac{d \xi_{j}}{\lambda}
$$

Since also

$$
\frac{1}{\xi_{0}^{1 / 2}}=\left\{\frac{i P(t) \pm \Phi(t)}{\Phi^{2 / 3}}\right\}^{1 / 2} \frac{\lambda^{1 / 6}}{\xi_{j}^{1 / 2}}
$$

we see that

$$
\frac{\lambda^{1 / 3} d t}{\left[\xi_{0}^{2}(t)\right]^{2}}=\left\{\frac{p_{1,0}+i \phi}{i p_{2,0}}\right\}\left\{\frac{\xi_{0}^{1 / 6}}{\xi_{0}^{8}}\right\}^{2}\left\{\frac{i P \pm \Phi}{\Phi^{2 / 3}}\right\}^{1 / 2} \frac{d \xi_{j}}{\lambda^{1 / 2} \xi_{j}^{1 / 2}} .
$$

The functions within braces are all bounded. Hence the integral (12.2) is a bounded multiple of

$$
\int_{\Gamma_{j, \mu}}\left|\frac{d \xi_{j}}{\lambda^{1 / 2} \xi_{j}^{1 / 2}}\right| .
$$

The boundedness of this can be established by the reasoning applied to the case $j=0$.

Corollary. An integral of the form

$$
\int_{\Gamma_{j, \mu}}\left|B(z, t, \lambda) \frac{\lambda^{1 / 6} d t}{\xi_{0}^{z}(t)}\right|
$$

is bounded, uniformly in $\mathrm{z}$, when $|\lambda|>N$.

Since $\xi_{0}^{g}(t) / \lambda^{1 / 6}$ is bounded, this integral is in fact of the form (12.2).

13. A solution $u_{k, 0}(z)$. When $\lambda$ and $z$ are confined to a pair of associated regions, the variable $\xi_{0}$ is confined to a region that lies in some sector (8.1). If it lies in just one such sector the integer $k$ is thereby determined. If it lies in each of two consecutive sectors (8.1), as it may, $k$ may be chosen as either one of the relevant integers, for the results to be obtained from the alternative choices are then asymptotically equivalent for the $\lambda$ and $z$ in question.

Let the functions $Y_{j, 0}(z)$ be defined by the formulas

$$
\begin{aligned}
& Y_{0,0}(z)=\lambda^{r-2-1 / 6} \xi_{0}^{\prime}(z) Q\left(y_{0}, z\right), \\
& Y_{j, 0}(z)=\lambda^{r-2} \xi_{0}^{\prime}(z) e^{-z \xi_{j}^{(z)}} Q\left(y_{k, j}, z\right), \quad j=1,2 .
\end{aligned}
$$

It follows from (9.8) that these functions are bounded, namely that with a suitable constant $M$

$$
\left|Y_{3,0}(z)\right|<M, \quad j=0,1,2,
$$

when $|\lambda|>N$. 
Consider the equation (10.3) when the lower limits of integration are taken at $z_{2, m}, z_{1, m}$, and $z_{2, m}$ respectively, and the constants are given the values $c_{0}=1, c_{1}=c_{2}=0$. If the $f(z)$ is then specifically denoted by $f_{0}(z)$, the equation is

$$
f_{0}(z)=Q\left(y_{0}, z\right)\left[1+\int_{\Gamma_{2, m}} \Omega_{0}(t) f_{0}(t) d t\right]+\sum_{j=1}^{2} Q\left(y_{k, j}, z\right) \int_{\Gamma_{j, m}} \Omega_{j}(t) f_{0}(t) d t .
$$

Let this be multiplied by $\lambda^{r-2-1 / 6} \xi_{0}^{s}(z)$. It can then be written as

$$
F_{0}(z)=Y_{0,0}(z)+\frac{1}{\lambda^{r}} \int_{\Gamma_{1, m}+\Gamma_{2, m}} K_{0}(z, t, \lambda) F_{0}(t) d t,
$$

with

$$
F_{0}(z)=\lambda^{r-2-1 / 6} \xi_{0}^{\prime}(z) f_{0}(z),
$$

and

$$
\text { (13.5) } \quad K_{0}(z, t, \lambda)=\left\{\begin{array}{l}
\frac{\lambda^{2} Y_{1,0}(z) e^{i \xi_{1}(z)} \Omega_{1}(t)}{\xi_{0}^{s}(t)}, \text { on } \Gamma_{1, m}, \\
\frac{\lambda^{2+1 / 6} Y_{0,0}(z) \Omega_{0}(t)}{\xi_{0}^{s}(t)}+\frac{\lambda^{2} Y_{2,0}(z) e^{i \xi_{2}(z)} \Omega_{2}(t)}{\xi_{0}^{z}(t)}, \text { on } \Gamma_{2, m} .
\end{array}\right.
$$

The relation (13.3) is an integral equation. Its kernel (13.5) has, by (9.7) and (13.2), the form

$$
K_{0}(z, t, \lambda)=\left\{\begin{array}{l}
\frac{\lambda^{1 / 3} e^{i \xi_{1}(z)-i \xi_{1}(t)} B(z, t, \lambda)}{\left[\xi_{0}^{g}(t)\right]^{2}}, \text { on } \Gamma_{1, m}, \\
\frac{\lambda^{1 / 6} B(z, t, \lambda)}{\xi_{0}^{8}(t)}+\frac{\lambda^{1 / 3} e^{i \xi_{2}(z)-i \xi_{2}(t)} B(z, t, \lambda)}{\left[\xi_{0}^{g}(t)\right]^{2}}, \text { on } \Gamma_{2, m} .
\end{array}\right.
$$

The exponentials in this are all bounded on the respective paths, by (12.1). Hence the respective integrals of $\left|K_{0}(z, t, \lambda) d t\right|$ are of the type (12.2). By the lemma of $\$ 12$, therefore, there is a constant $M_{0}$ for which

$$
\int_{\Gamma_{1, m}+\Gamma_{2, m}}\left|K_{0}(z, t, \lambda) d t\right|<M_{0} .
$$

The relation

$$
F_{0}(z)=\sum_{n=0}^{\infty}\left(\frac{1}{\lambda^{r}}\right)^{n} Y_{0, n}(z)
$$

in which 


$$
Y_{0, n}(z)=\int_{\Gamma_{1, m}+\Gamma_{2, m}} K_{0}(z, t, \lambda) Y_{0, n-1}(t) d t, \quad n=1,2,3, \cdots .
$$

is formally obtainable by the repeated iteration of the equation (13.3). It yields a solution of that equation when the series involved in it converges uniformly. Now because of (13.2) and (13.6) an induction upon the basis of (13.8) shows that

$$
\left|Y_{0, n}(z)\right|<M M_{0}^{n} .
$$

Hence when $|\lambda|>N$ the series in (13.7) does converge uniformly. $F_{0}(z)$ accordingly exists and is bounded, and it follows from this by (13.4) that

$$
f_{0}(z)=\frac{B(z, \lambda)}{\lambda^{r-2-1 / 6} \xi_{0}^{s}(z)} .
$$

Following the argument set forth in $\$ 10$, we now define $u_{k, 0}(z)$ by the formula (10.1) with the same lower limits of integration and constants as were used above. This and its derived formulas (10.2) are specifically

$$
\begin{aligned}
& u_{k, 0}(z)=y_{k, 0}(z)+\sum_{n=0}^{2} \alpha_{0, n}(z) y_{k, n}(z), \\
& u_{k, 0}^{\prime}(z)=y_{k, 0}^{\prime}(z)+\sum_{n=0}^{2} \alpha_{0, n}(z) y_{k, n}^{\prime}(z), \\
& u_{k, 0}^{\prime \prime}(z)=y_{k, 0}^{\prime \prime}(z)+\sum_{n=0}^{2} \alpha_{0, n}(z) y_{k, n}^{\prime \prime}(z),
\end{aligned}
$$

with

$$
\alpha_{0,0}(z)=\int_{\Gamma_{2, m}} \Omega_{0}(t) f_{0}(t) d t, \quad \alpha_{0, j}(z)=\int_{\Gamma_{j, m}} \Omega_{j}(t) f_{0}(t) d t, \quad j=1,2 .
$$

The evaluations (9.7) and (13.9) show that

$$
\begin{aligned}
& \alpha_{0,0}(z)=\frac{1}{\lambda^{r}} \int_{\Gamma_{2, m}} \frac{\lambda^{1 / 6} B(t, \lambda)}{\xi_{0}^{s}(t)} d t, \\
& \alpha_{0, j}(z)=\frac{\lambda^{1 / 6} e^{-i \xi_{j}(z)}}{\lambda^{r}} \int_{\Gamma_{j, m}} \frac{\lambda^{1 / 3} e^{i \xi_{j}(z)-i \xi_{j}(t)} B(t, \lambda)}{\left[\xi_{0}^{\xi}(t)\right]^{2}} d t .
\end{aligned}
$$

By the lemma of $\$ 12$ it follows that

$$
\begin{aligned}
& \alpha_{0,0}(z)=\frac{B(z, \lambda)}{\lambda^{r}}, \\
& \alpha_{0, j}(z)=\frac{\lambda^{1 / 6} e^{-i \xi_{j(z)}} B(z, \lambda)}{\lambda^{r}},
\end{aligned}
$$

The relations (9.5), (9.6), and (13.11), as applied to (13.10), now yield the 
following result, that while $\lambda$ and $z$ remain in their original associated regions

$$
\begin{aligned}
& u_{k, 0}(z)=y_{k, 0}(z)+\frac{\lambda^{1 / 6} B(z, \lambda)}{\lambda^{r} \xi_{0}^{s}(z)}, \\
& u_{k, 0}^{\prime}(z)=y_{k, 0}^{\prime}(z)+\frac{\lambda^{1 / 6} B(z, \lambda)}{\lambda^{r-1} \xi_{0}^{s}(z)}, \\
& u_{k, 0}^{\prime \prime}(z)=y_{k, 0}^{\prime \prime}(z)+\frac{\lambda^{1 / 6} B(z, \lambda)}{\lambda^{r-2} \xi_{0}^{s}(z)} .
\end{aligned}
$$

Finally, it may be seen from (13.10), since $L\left(y_{k, j}\right)=0$ for $j=1,2$, that $L\left(u_{k, 0}\right)=L\left(y_{0}\right)\left\{1+\alpha_{0,0}(z)\right\}$. Hence, by (6.3) and (13.11),

$$
L\left(u_{k, 0}\right)=\lambda^{2}\left\{1+\frac{B(z, \lambda)}{\lambda^{r}}\right\} .
$$

Since every z-region of an associated pair includes the origin upon its boundary, the evaluations (13.12) and (13.13) are valid in every case when $z=0$. The values of $y_{k, 0}(z)$ and its derivatives are obtainable from the formula (6.1).

It should be observed that different pairs of associated $\lambda$ and $z$-regions may all yield $\xi_{0}$-regions that lie within a single sector $\boldsymbol{\Xi}_{k}$, and may therefore have the same value of $k$ associated with them. Within each of these pairs of regions some solution has the form (13.12). The solution $u_{k, 0}(z)$ for one such pair of regions need not, however, be the same as the solution $u_{k, 0}(z)$ for another pair.

14. The solutions $u_{k, j}(z)$, for $j=1,2$. The procedure of $\$ 13$ leads also to the forms of a further pair of solutions. Let $f(z)$ be designated by $f_{1}(z)$ when the equation (10.3) is taken with the lower limits of integration at $z_{1, M}$, $z_{0, M}$, and $z_{0, M}$ respectively, and the constants are given the values $c_{0}=0$, $c_{1}=1, c_{2}=0$. Then, after it has been multiplied by $\lambda^{-2} \xi_{0}^{s}(z) e^{-i \xi_{1}(z)}$, the equation can be written as

with

$$
F_{1}(z)=Y_{1,0}(z)+\frac{1}{\lambda^{r}} \int_{\Gamma_{0, M}+\Gamma_{1, M}} K_{1}(z, t, \lambda) F_{1}(t) d t,
$$

and

$$
K_{1}(z, t, \lambda)=\left\{\begin{array}{l}
\frac{\lambda^{2} Y_{1,0}(z) \Omega_{1}(t) e^{i \xi_{1}(t)}}{\xi_{0}^{z}(t)}+\frac{\lambda^{2} Y_{2,0}(z) e^{-2 i \xi_{0}(z)+i \xi_{1}(t)} \Omega_{2}(t)}{\xi_{0}^{s}(t)}, \text { on } \Gamma_{0, M}, \\
\frac{\lambda^{2+1 / 6} Y_{0,0}(z) e^{-i \xi_{1}(z)+i \xi_{1}(t)} \Omega_{0}(t)}{\xi_{0}^{z}(t)}, \text { on } \Gamma_{1, M .} .
\end{array}\right.
$$

By virtue of the evaluations (9.7) we see that 


$$
K_{1}(z, t, \lambda)= \begin{cases}\frac{\lambda^{1 / 3} B(z, t, \lambda)}{\left[\xi_{0}^{s}(t)\right]^{2}}+\frac{\lambda^{1 / 3} e^{-2 i \xi_{0}(z)+2 i \xi_{0}(t)} B(z, t, \lambda)}{\left[\xi_{0}^{s}(t)\right]^{2}}, \text { on } \Gamma_{0, M}, \\ \frac{\lambda^{1 / 6} e^{-i \xi_{1}(z)+i \xi_{1}(t)} B(z, t, \lambda)}{\xi_{0}^{s}(t)}, \text { on } \Gamma_{1, M .} .\end{cases}
$$

The exponentials here involved are bounded on the respective paths, and, because the integrals are of the forms (12.2) or (12.4), it follows that

$$
\int_{\Gamma_{0, M}+\Gamma_{1, M}}\left|K_{1}(z, t, \lambda) d t\right|<M_{1}
$$

with some constant $M_{1}$. This relation, together with (13.2), insures the uniform convergence of the series that results from the repeated iteration of the equation (14.1). Therefore $F_{1}(z)$ exists and is bounded, and, by (14.2),

$$
f_{1}(z)=\frac{e^{i \xi_{1}(z)} B(z, \lambda)}{\lambda^{r-2} \xi_{0}^{s}(z)} .
$$

A solution $u_{k, 1}(z)$ and its derivatives are now given by the formulas (10.1) and (10.2), namely

$$
\begin{aligned}
& u_{k, 1}(z)=y_{k, 1}(z)+\sum_{n=0}^{2} \alpha_{1, n}(z) y_{k, n}(z), \\
& u_{k, 1}^{\prime}(z)=y_{k, 1}^{\prime}(z)+\sum_{n=0}^{2} \alpha_{1, n}(z) y_{k, n}^{\prime}(z), \\
& u_{k, 1}^{\prime \prime}(z)=y_{k, 1}^{\prime \prime}(z)+\sum_{n=0}^{2} \alpha_{1, n}(z) y_{k, n}^{\prime \prime}(z),
\end{aligned}
$$

with

$$
\alpha_{1,0}(z)=\int_{\Gamma_{1, M}} \Omega_{0}(t) f_{1}(t) d t, \quad \alpha_{1, j}(z)=\int_{\Gamma_{0, M}} \Omega_{j}(t) f_{1}(t) d t, \quad j=1,2 .
$$

By virtue of the evaluations (9.7) and (14.3), and the lemma of $\$ 12$, it follows that

$$
\begin{aligned}
& \alpha_{1,0}(z)=\frac{e^{i \xi_{1}(z)} B(z, \lambda)}{\lambda^{r+1 / 6}}, \\
& \alpha_{1,1}(z)=\frac{B(z, \lambda)}{\lambda^{r}}, \\
& \alpha_{1,2}(z)=\frac{e^{2 i \xi_{0}(z)} B(z, \lambda)}{\lambda^{r}}
\end{aligned}
$$

Therewith the relations (14.4) yield the forms 


$$
\begin{aligned}
& u_{k, 1}(z)=y_{k, 1}(z)+\frac{e^{i \xi_{1}(z)} B(z, \lambda)}{\lambda^{r} \xi_{0}^{\prime}(z)}, \\
& u_{k, 1}^{\prime}(z)=y_{k, 1}^{\prime}(z)+\frac{e^{i \xi_{1}(z)} B(z, \lambda)}{\lambda^{r-2} \xi_{0}^{z}(z)}, \\
& u_{k, 1}^{\prime \prime}(z)=y_{k, 1}^{\prime \prime}(z)+\frac{e^{i \xi_{1}(z)} B(z, \lambda)}{\lambda^{r-1} \xi_{0}^{\prime}(z)} .
\end{aligned}
$$

From (14.4) and (14.5) it is also to be seen that

$$
L\left(u_{k, 1}\right)=\lambda^{2} e^{i \xi_{1}(z)} B(z, \lambda) / \lambda^{r+1 / 6} .
$$

Let $f(z)$ be designated by $f_{2}(z)$ when the equation (10.3) is taken with the lower limits of integration at $z_{2, M}, z_{0, m}$, and $z_{0, m}$ respectively, and the constants are given the values $c_{0}=c_{1}=0, c_{2}=1$. The equation, after it has been multiplied by $\lambda^{r-2} \xi_{0}(z) e^{-i \xi_{2}(z)}$, may then be written as

$$
\begin{gathered}
F_{2}(z)=Y_{2,0}(z)+\frac{1}{\lambda^{r}} \int_{\Gamma_{0, m}+\Gamma_{2, \mu}} K_{2}(z, t, \lambda) F_{2}(t) d t, \\
\text { with } F_{2}(z)=\lambda^{r-2} \xi_{0}^{\prime}(z) e^{-i \xi_{2}(z)} f_{2}(z), \text { and } \\
K_{2}(z, t, \lambda)=\left\{\begin{array}{l}
\frac{\lambda^{2} Y_{1,0}(z) e^{2 i \xi_{0}(z)+i \xi_{2}(t)} \Omega_{1}(t)}{\xi_{0}^{s}(t)}+\frac{\lambda^{2} Y_{2,0}(z) e^{i \xi_{2}(t)} \Omega_{2}(t)}{\xi_{0}^{s}(t)}, \text { on } \Gamma_{0, m}, \\
\frac{\lambda^{2+1 / 6} Y_{0,0}(z) e^{-i \xi_{2}(z)+i \xi_{2}(t)} \Omega_{0}(t)}{\xi_{0}^{s}(t)}, \text { on } \Gamma_{2, M} .
\end{array}\right.
\end{gathered}
$$

From this we find in the manner now familiar that

$$
f_{2}(z)=e^{i \xi_{2}(z)} B(z, \lambda) / \lambda^{-2} \xi_{0}^{o}(z),
$$

and that in the relation

$$
u_{k, 2}(z)=y_{k, 2}(z)+\sum_{n=0}^{2} \alpha_{2, n}(z) y_{k, n}(z),
$$

the coefficients $\alpha_{2, n}(z)$ are of the forms

$$
\begin{aligned}
& \alpha_{2,0}(z)=\frac{e^{i \xi_{2}(z)} B(z, \lambda)}{\lambda^{r+1 / 6}}, \\
& \alpha_{2,1}(z)=\frac{e^{-2 i \xi_{0}(z)} B(z, \lambda)}{\lambda^{r}}, \\
& \alpha_{2,2}(z)=\frac{B(z, \lambda)}{\lambda^{r}}
\end{aligned}
$$


The equation (14.8) and its derivatives therefore yield the forms

$$
\begin{aligned}
& u_{k, 2}(z)=y_{k, 2}(z)+\frac{e^{i \xi_{2}(z)} B(z, \lambda)}{\lambda^{r} \xi_{0}^{g}(z)}, \\
& u_{k, 2}^{\prime}(z)=y_{k, 2}^{\prime}(z)+\frac{e^{i \xi_{2}(z)} B(z, \lambda)}{\lambda^{r-1} \xi_{0}^{s}(z)}, \\
& u_{k, 2}^{\prime \prime}(z)=y_{k, 2}^{\prime \prime}(z)+\frac{e^{i \xi_{2}(z)} B(z, \lambda)}{\lambda^{r-2} \xi_{0}^{s}(z)},
\end{aligned}
$$

and

$$
L\left(u_{k, 2}\right)=\frac{\lambda^{2} e^{i \xi_{2}(z)} B(z, \lambda)}{\lambda^{r+1 / 6}} .
$$

The representations (14.6) and (14.10) maintain, like (13.12), in a pair of associated regions for which $\xi_{0}$ lies in the sector $\Xi_{k}$. They are, in every case, valid at $z=0$. The values of $y_{k, j}$ and their derivatives at $z=0$ may be obtained from the relations (8.9) and (7.6).

15. The general solution identified at $z=0$. Since $z=0$ is an ordinary (nonsingular) point of the given differential equation, any solution $u(z)$ is identified by its values

$$
u(0), \quad u^{\prime}(0), \quad u^{\prime \prime}(0) .
$$

When any $\lambda$ for which $|\lambda|>N$, and any $z$ in $R(z)$ are given, the sector (8.1) which contains $\xi_{0}$ is identified. For these values of $\lambda$ and $z$ the solutions $u_{k, j}(z), j=0,1,2$, accordingly have the forms given by the formulas (13.12), (14.6), and (14.10). Now the right-hand member of the relation

$$
u(z)=\frac{1}{W\left(u_{k, 0}, u_{k, 1}, u_{k, 2} ; 0\right)}\left|\begin{array}{llll}
u_{k, 0}(z) & u_{k, 1}(z) & u_{k, 2}(z) & 0 \\
u_{k, 0}(0) & u_{k, 1}(0) & u_{k, 2}(0) & u(0) \\
u_{k, 0}^{\prime}(0) & u_{k, 1}^{\prime}(0) & u_{k, 2}^{\prime}(0) & u^{\prime}(0) \\
u_{k, 0}^{\prime \prime}(0) & u_{k, 1}^{\prime \prime}(0) & u_{k, 2}^{\prime \prime}(0) & u^{\prime \prime}(0)
\end{array}\right|
$$

is a linear combination of the functions $u_{k, j}(z)$, and is therefore a solution. It is readily seen to assume the values (15.1), whereby the formula (15.2) is established.

For the Wronskian that appears in this formula the relations (13.10), (14.4), and (14.8) yield

$$
W\left(u_{k, 0}, u_{k, 1}, u_{k, 2} ; z\right)=W\left(y_{k, 0}, y_{k, 1}, y_{k, 2} ; z\right) \Delta(z),
$$

with

$$
\Delta(z)=\left|\begin{array}{ccc}
1+\alpha_{0,0}(z) & \alpha_{1,0}(z) & \alpha_{2,0}(z) \\
\alpha_{0,1}(z) & 1+\alpha_{1,1}(z) & \alpha_{2,1}(z) \\
\alpha_{0,2}(z) & \alpha_{1,2}(z) & 1+\alpha_{2,2}(z)
\end{array}\right|
$$


From the evaluations (13.11), (14.5), and (14.9) it follows that

$$
\Delta(z)=1+B(z, \lambda) / \lambda^{r} .
$$

This, taken with (8.12), yields the result

$$
W\left(u_{k, 0}, u_{k, 1}, u_{k, 2} ; z\right)=-2 i \lambda^{8 / 3} \exp \left(-2 \lambda \int_{0}^{z} p_{1}(z, \lambda) d r\right)\left\{1+\frac{B(\lambda)}{\lambda^{r}}\right\}
$$

In this we have written $B(\lambda)$ instead of $B(z, \lambda)$, since a dependence of this function upon $z$ is precluded by the form of the given differential equation (2.3). The formula (15.2) is thus more explicitly

$$
u(z)=\frac{i}{2 \lambda^{8 / 3}}\left\{1+\frac{B(\lambda)}{\lambda^{r}}\right\}\left|\begin{array}{cccc}
u_{k, 0}(z) & u_{k, 1}(z) & u_{k, 2}(z) & 0 \\
u_{k, 0}(0) & u_{k, 1}(0) & u_{k, 2}(0) & u(0) \\
u_{k, 0}^{\prime}(0) & u_{k, 1}^{\prime}(0) & u_{k, 2}^{\prime}(0) & u^{\prime}(0) \\
u_{k, 0}^{\prime \prime}(0) & u_{k, 1}^{\prime \prime}(0) & u_{k, 2}^{\prime \prime}(0) & u^{\prime \prime}(0)
\end{array}\right| .
$$

The relation (15.6) is of the form

$$
u(z)=C_{0}(\lambda) u_{k, 0}(z)+C_{1}(\lambda) u_{k, 1}(z)+C_{2}(\lambda) u_{k, 2}(z),
$$

with coefficients $C_{j}(\lambda)$ that are explicitly determinable to the terms in $1 / \lambda^{r}$. To that extent the form of $u(z)$ is thus given when $z$ and $\lambda$ are such that $\xi_{0}$ lies in the sector $\boldsymbol{\Xi}_{k}$. This result remains deficient in the case that the explicit part of the coefficient of the dominant member of the set $u_{k, j}(z)$ turns out to be zero, for it then remains ambiguous whether this coefficient is nonvanishing of the order of $1 / \lambda^{m}$ with some $m>r$, or whether it is actually zero. This ambiguity is inherent in asymptotic representation. It is because of this that the forms for the solutions $u_{k, j}(z)$ for different indices $k$, not merely for a single $k$, have been determined. Within the limitation described, the formula (15.6) can, of course, be applied to any solution $u_{k, j}(z)$ with any index $k$, since the values of this solution at $z=0$ have been asymptotically determined.

16. A comparison with the case of no turning point. In any closed bounded $z$-region in which the roots of the auxiliary equation (1.3) are distinct, and hence, in particular, in any closed part of our region $R(z)$ that does not include the turning point, classical procedures may be applied to derive the forms of the solutions of the given differential equation (1.1). By substituting for $w$ an expression

$$
\exp \left(\lambda \int \chi_{j}(z) d r\right) \sum_{n=0}^{\infty} \frac{s_{j, n}(z)}{\lambda^{n}},
$$

in which $\chi_{j}(z)$ is any root of (1.3), and the coefficients $s_{j, n}(z)$ are undetermined, the differential equation is given the form 


$$
\exp \left(\lambda \int \chi_{j}(z) d r\right) \lambda^{2} \sum_{n=0}^{\infty} \frac{S_{j, n}(z)}{\lambda^{n}}=0 .
$$

In this the functions $S_{j, n}(z)$ are all expressible in terms of the coefficients $s_{j, n}(z)$, the leading one being

$$
S_{j, 0}(z) \equiv \theta_{0, j}(z) s_{j, 0}^{\prime}+\theta_{1, j}(z) s_{j, 0},
$$

with

$$
\begin{aligned}
& \dot{\theta_{0, j}}=3 \chi_{j}^{2}+2 h_{1,0} \chi_{j}+h_{2,0}, \\
& \theta_{1, j}=\left[3 \chi_{j}+h_{1,0}\right] \chi_{j}^{\prime}+h_{1,1} \chi_{j}^{2}+h_{2,1} \chi_{j}+h_{3,1} .
\end{aligned}
$$

For $n=1,2,3, \cdots$, it is found that

$$
S_{j, n}(z)=\theta_{0, j} s_{j, n}^{\prime}+\theta_{1, j} s_{j, n}+f_{j, n}(z),
$$

with $f_{j, n}$ standing for a function that involves the coefficients $s_{j, m}$ only for $m<n$.

The equation (16.2) is formally fulfilled if each function $S_{j, n}(z)$ is identically zero. This can be assured by choosing $s_{j, 0}(z)$ as a solution of the differential equation

$$
\theta_{0, j}(z) s_{j, 0}^{\prime}+\theta_{1, j}(z) s_{j, 0}=0,
$$

and then choosing the $s_{j, n}(z)$ for $n=1,2,3, \cdots$, in turn as solutions of the respective differential equations

$$
\theta_{0, j}(z) s_{j, n}^{\prime}+\theta_{1, j}(z) s_{j, n}+f_{j, n}(z)=0 .
$$

Although the expression (16.1) thus obtained formally fulfills the differential equation, it fails to give a solution, because the series involved in it diverges. However, it can be shown that in appropriately defined sub-regions of $\lambda$ and $z$ there are solutions $w_{j}(z)$ that have the forms

$$
w_{j}(z)=\exp \left(\lambda \int \chi_{j}(z) d z\right)\left\{\sum_{n=0}^{r-1} \frac{s_{j, n}(z)}{\lambda^{n}}+\frac{B(z, \lambda)}{\lambda^{r}}\right\}, \quad j=0,1,2 .
$$

If, before this deduction is applied, the given differential equation is put into the normal form (2.12), the roots of its auxiliary equation are explicitly $\chi_{0}(z) \equiv 0, \chi_{j}(z)=-p_{1,0}(z) \pm i \phi(z), j=1,2$. The forms (16.6) are then

$$
\begin{aligned}
& u_{0}(z)=\sum_{n=0}^{r-1} \frac{s_{0, n}(z)}{\lambda^{n}}+\frac{B(z, \lambda)}{\lambda^{r}}, \\
& u_{j}(z)=e^{i \xi_{j}} \sum_{n=0}^{r-1} \frac{s_{j, n}(z)}{\lambda^{n}}+\frac{e^{i \xi_{j}} B(z, \lambda)}{\lambda^{r}}, \quad j=1,2 .
\end{aligned}
$$


These are the analogues of the forms which, in the presence of a turning point, we have derived as (13.12), (14.6), and (14.10).

The way in which the classical procedure fails when the $z$-region contains a turning point is now readily discerned. A turning point is by definition one in which a multiplicity among the roots of the auxiliary equation occurs. It is therefore a point in which at least two of the functions $\theta_{0, j}(z)$, as given by (16.3), vanish. The differential equations (16.4) and (16.5), from which the coefficients of the forms (16.6) or (16.7) must be determined, are therefore singular at this point. Hence the coefficients $s_{j, n}(z)$, and therewith the whole explicit parts of the formulas (16.7), become infinitely discontinuous. Near a turning point, therefore, the forms yielded by the classical theory cannot be used.

\section{REFERENCES}

1. R. E. Langer, The boundary problem of an ordinary linear differential system in the complex domain, Trans. Amer. Math. Soc. vol. 46 (1939) pp. 151-190.

2. H. L. Turrittin, Asymptotic solutions of certain ordinary differential equations associated with multiple roots of the characteristic equation, Amer. J. Math. vol. 58 (1936) pp. 364-376.

3. W. Wasow, The complex asymptotic theory of a fourth order differential equation of hydrodynamics, Ann. of Math. vol. 49 (1948) pp. 852-871.

4. R. E. Langer, Asymptotic solutions of a differential equation in the theory of microwave propagation, Communications on Pure and Applied Mathematics vol. 3 (1950) pp. 427-438.

5. W. Wasow, $A$ study of the solutions of the differential equation $y^{\text {iv }}+\lambda^{2}\left(x y^{\prime \prime}+y\right)=0$, for large values of $\lambda$, Ann. of Math. vol. 52 (1950) pp. 350-361.

6. D. Meksyn, Asymptotic integrals of a fourth order differential equation containing a large parameter, Proc. London Math. Soc. (2) vol. 49 (1947) pp. 436-457.

7. - Stability of viscous flow between rotating cylinders, Proc. Roy. Soc. London Ser. A vol. 187 (1946) pp. 115-128, 480-504.

8. R. E. Langer, The asymptotic solutions of ordinary linear differential equations of the second order with special reference to a turning point, Trans. Amer. Math. Soc. vol. 67 (1949) pp. 461-490. 1944.

9. G. N. Watson, A treatise on the theory of Bessel functions, 2d ed., Cambridge, New York,

The University OF Wisconsin, MADISON, WIS. 